CUBO A Mathematical Journal

Vol.22, $N^{\underline{O}} 01$, (23-37). April 2020

\title{
ฤ-Ricci Solitons on 3-dimensional Trans-Sasakian Manifolds
}

\author{
SAMPA PAHAN \\ Department of Mathematics, \\ Mrinalini Datta Mahavidyapith \\ Kolkata-700051, India. \\ sampapahan25@gmail.com
}

\begin{abstract}
In this paper, we study $\eta$-Ricci solitons on 3-dimensional trans-Sasakian manifolds. Firstly we give conditions for the existence of these geometric structures and then observe that they provide examples of $\eta$-Einstein manifolds. In the case of $\phi$-Ricci symmetric trans-Sasakian manifolds, the $\eta$-Ricci soliton condition turns them to Einstein manifolds. Afterward, we study the implications in this geometric context of the important tensorial conditions $R \cdot S=0, S \cdot R=0, W_{2} \cdot S=0$ and $S \cdot W_{2}=0$.
\end{abstract}

\section{RESUMEN}

En este artículo estudiamos solitones $\eta$-Ricci en variedades trans-Sasakianas tridimensionales. En primer lugar damos condiciones para la existencia de estas estructuras geométricas y luego observamos que ellas dan ejemplos de variedades $\eta$-Einstein. En el caso de variedades trans-Sasakianas $\phi$-Ricci simétricas, la condición de solitón $\eta$-Ricci las convierte en variedades Einstein. A continuación estudiamos las implicancias en este contexto geométrico de las importantes condiciones tensoriales $\mathrm{R} \cdot \mathrm{S}=0, \mathrm{~S} \cdot \mathrm{R}=0$, $\mathrm{W}_{2} \cdot \mathrm{S}=0$ y $\mathrm{S} \cdot \mathrm{W}_{2}=0$.

Keywords and Phrases: Trans-Sasakian manifold, ๆ-Ricci solitons.

2010 AMS Mathematics Subject Classification: 53C21, 53C25, 53C44. 


\section{Introduction}

In 1982, the notion of the Ricci flow was introduced by Hamilton 10 to find a canonical metric on a smooth manifold.The Ricci flow is an evolution equation for Riemannian metric $g(t)$ on a smooth manifold $M$ given by

$$
\frac{\partial}{\partial t} g(t)=-2 S
$$

A solution to this equation (or a Ricci flow) is a one-parameter family of metrics $g(t)$, parameterized by $t$ in a non-degenerate interval $I$, on a smooth manifold $M$ satisfying the Ricci flow equation. If I has an initial point $t_{0}$, then $\left(M, g\left(t_{0}\right)\right)$ is called the initial condition of or the initial metric for the Ricci flow (or of the solution) [14].

Ricci solitons and $\eta$-Ricci solitons are natural generalizations of Einstein metrics. A Ricci soliton on a Riemannian manifold $(M, g)$ is defined by

$$
S+\frac{1}{2} \mathcal{L} \times g=\lambda g
$$

where $\mathcal{L}_{X} \mathrm{~g}$ is the Lie derivative along the vector field $\mathrm{X}, \mathrm{S}$ is the Ricci tensor of the metric and $\lambda$ is a real constant. If $X=\nabla f$ for some function $f$ on $M$, the Ricci soliton becomes gradient Ricci soliton. Ricci solitons appear as self-similar solutions to Hamiltons's Ricci flow and often arise as limits of dilations of singularities in the Ricci flow [11. A soliton is called shrinking, steady and expanding according as $\lambda>0, \lambda=0$ and $\lambda<0$ respectively.

In 2009, the notion of $\eta$-Ricci soliton was introduced by J.C. Cho and M. Kimura [6]. J.C. Cho and M. Kimura proved that a real hypersurface admitting an $\eta$-Ricci soliton in a non-flat complex space form is a Hopf-hypersurface [6]. An $\eta$-Ricci soliton on a Riemannian manifold $(M, g)$ is defined by the following equation

$$
2 S+\mathcal{L}_{\xi} g+2 \lambda g+2 \mu \eta \otimes \eta=0,
$$

where $\mathcal{L}_{\xi}$ is the Lie derivative operator along the vector field $\xi, S$ is the Ricci tensor of the metric and $\lambda, \mu$ are real constants. If $\mu=0$, then $\eta$-Ricci soliton becomes Ricci soliton.

In the last few years, many authors have worked on Ricci solitons and their generalizations in different Contact metric manfolds in [1, [7, [8, [9], [12] etc. In 2014, B. Y. Chen and S. Deshmukh have established the characterizations of compact shrinking trivial Ricci solitons in [5]. Also, in [2, A. Bhattacharyya, T. Dutta, and S. Pahan studied the torqued vector field and established some applications of torqued vector field on Ricci soliton and conformal Ricci soliton. A.M. Blaga [3], D. G. Prakasha and B. S. Hadimani [17] observed $\eta$-Ricci solitons on different contact metric manifolds satisfying some certain curvature conditions. 
In this paper we study the existence of $\eta$-Ricci soliton on 3-dimensional trans-Sasakian manifold. Next we show that $\eta$-Ricci soliton on 3-dimensional trans-Sasakian manifolds becomes $\eta$-Einstein Manifold under some conditions. Next we prove that $\phi$-Ricci symmetric trans-Sasakian manifold $(M, g)$ manifold satisfying an $\eta$-Ricci soliton becomes an Einstein manifold. Next we give an example of an $\eta$-Ricci soliton on 3-dimensional trans-Sasaian manifold with $\lambda=-2$ and $\mu=6$. Later we obtain some different types of curvature tensors and their properties under certain conditions.

\section{Preliminaries}

The product $\bar{M}=M \times R$ has a natural almost complex structure J with the product metric $G$ being Hermitian metric. The geometry of the almost Hermitian manifold $(\bar{M}, J, G)$ gives the geometry of the almost contact metric manifold $(M, \phi, \xi, \eta, g)$. Sixteen different types of structures on $M$ like Sasakian manifold, Kenmotsu manifold etc are given by the almost Hermitian manifold ( $\bar{M}, J, G)$. The notion of trans-Sasakian manifolds was introduced by Oubina [15] in 1985. Then J. C. Marrero 13 have studied the local structure of trans-Sasakian manifolds. In general a trans-Sasakian manifold $(M, \phi, \xi, \eta, g, \alpha, \beta)$ is called a trans-Sasakian manifold of type $(\alpha, \beta)$. An $n(=2 m+1)$ dimensional Riemannian manifold $(M, g)$ is called an almost contact manifold if there exists a $(1,1)$ tensor field $\phi$, a vector field $\xi$ and a 1-form $\eta$ on $M$ such that

$$
\begin{gathered}
\phi^{2}(X)=-X+\eta(X) \xi, \\
\eta(\xi)=1, \eta(\phi X)=0, \\
\phi \xi=0, \\
\eta(X)=g(X, \xi), \\
g(\phi X, \phi Y)=g(X, Y)-\eta(X) \eta(Y), \\
g(X, \phi Y)+g(Y, \phi X)=0,
\end{gathered}
$$

for any vector fields $X, Y$ on $M$. A 3-dimensional almost contact metric manifold $M$ is called a trans-Sasakian manifold if it satisfies the following condition

$$
(\nabla \times \phi)(Y)=\alpha\{g(X, Y) \xi-\eta(Y) X\}+\beta\{g(\phi X, Y) \xi-\eta(Y) \phi X\}
$$

for some smooth functions $\alpha, \beta$ on $M$ and we say that the trans-Sasakian structure is of type $(\alpha, \beta)$. For 3-dimensional trans-Sasakian manifold, from (2.7) we have,

$$
\nabla_{X} \xi=-\alpha \phi X+\beta(X-\eta(X) \xi)
$$




$$
\left(\nabla_{X} \eta\right)(Y)=-\alpha g(\phi X, Y)+\beta g(\phi X, \phi Y)
$$

In a 3-dimensional trans-Sasakian manifold, we have

$$
\begin{aligned}
& R(X, Y) Z=\left[\frac{r}{2}-2\left(\alpha^{2}-\beta^{2}-\xi \beta\right)\right][g(Y, Z) X-g(X, Z) Y] \\
& -\left[\frac{r}{2}-3\left(\alpha^{2}-\beta^{2}\right)+\xi \beta\right][g(Y, Z) \eta(X)-g(X, Z) \eta(Y)] \xi \\
& +[g(Y, Z) \eta(X)-g(X, Z) \eta(Y)][\phi \operatorname{grad} \alpha-\operatorname{grad} \beta] \\
& -\left[\frac{r}{2}-3\left(\alpha^{2}-\beta^{2}\right)+\xi \beta\right] \eta(Z)[\eta(Y) X-\eta(X) Y] \\
& -[Z \beta+(\phi Z) \alpha] \eta(Z)[\eta(Y) X-\eta(X) Y] \\
& -[X \beta+(\phi X) \alpha][g(Y, Z) \xi-\eta(Z) Y] \\
& -[Y \beta+(\phi Y) \alpha][g(X, Z) \xi-\eta(Z) X], \\
& S(X, Y)=\left[\frac{r}{2}-\left(\alpha^{2}-\beta^{2}-\xi \beta\right)\right] g(X, Y) \\
& -\left[\frac{r}{2}-3\left(\alpha^{2}-\beta^{2}\right)+\xi \beta\right] \eta(X) \eta(Y) \\
& -[Y \beta+(\phi Y) \alpha] \eta(X)-[X \beta+(\phi X) \alpha] \eta(Y) \text {. }
\end{aligned}
$$

When $\alpha$ and $\beta$ are constants the above equations reduce to,

$$
\begin{gathered}
R(\xi, X) \xi=\left(\alpha^{2}-\beta^{2}\right)(\eta(X) \xi-X), \\
S(X, \xi)=2\left(\alpha^{2}-\beta^{2}\right) \eta(X), \\
R(\xi, X) Y=\left(\alpha^{2}-\beta^{2}\right)(g(X, Y) \xi-\eta(Y) X) . \\
R(X, Y) \xi=\left(\alpha^{2}-\beta^{2}\right)(\eta(Y) X-\eta(X) Y) .
\end{gathered}
$$

Definition 2.1. A trans-Sasakian manifold $M^{3}$ is said to be $\eta$-Einstein manifold if its Ricci tensor $\mathrm{S}$ is of the form

$$
S(X, Y)=a g(X, Y)+b \eta(X) \eta(Y),
$$

where $a, b$ are smooth functions. 


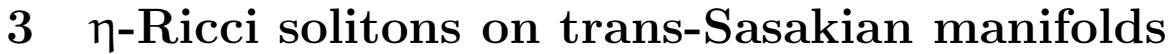

To study the existence conditions of $\eta$-Ricci solitons on 3-dimensional trans-Sasakian manifolds, we prove the following theorem.

Theorem 3.1: Let $(M, g, \phi, \eta, \xi, \alpha, \beta)$ be a 3-dimensional trans-Sasakian manifold with $\alpha, \beta$ constants $(\beta \neq 0)$. If the symmetric $(0,2)$ tensor field $h$ satisfying the condition $\beta h(X, Y)-$ $\frac{\alpha}{2}[h(\phi X, Y)+h(X, \phi Y)]=\mathcal{L}_{\xi} g(X, Y)+2 S(X, Y)+2 \mu \eta(X) \eta(Y)$ is parallel with respect to the LeviCivita connection associated to $g$. Then $(g, \xi, \mu)$ becomes an $\eta$-Ricci soliton.

Proof: We consider a symmetric $(0,2)$-tensor field $h$ which is parallel with respect to the LeviCivita connection $(\nabla h=0)$. Then it follows that

$$
h(R(X, Y) Z, W)+h(R(X, Y) Z, W)=0
$$

for an arbitary vector field $W, X, Y, Z$ on $M$. Put $X=Z=W=\xi$ we get

$$
h(R(X, Y) \xi, \xi)=0,
$$

for any $X, Y \in \chi(M)$ By using the equation (2.13)

$$
h(Y, \xi)=g(Y, \xi) h(\xi, \xi),
$$

for any $Y \in \chi(M)$. Differentiating the equation (3.3) covariantly with respect to the vector field $X \in \chi(M)$ we have

$$
h\left(\nabla_{X} Y, \xi\right)+h\left(Y, \nabla_{X} \xi\right)=g\left(\nabla_{X} Y, \xi\right) h(\xi, \xi)+g\left(Y, \nabla_{X} \xi\right) h(\xi, \xi),
$$

Using the equation (2.8) we have

$$
\beta h(X, Y)-\alpha h(\phi X, Y)=-\alpha g(\phi X, Y) h(\xi, \xi)+\beta h(\xi, \xi) g(X, Y) .
$$

Interchanging $X$ by $Y$ we have

$$
\beta h(X, Y)-\alpha h(X, \phi Y)=-\alpha g(X, \phi Y) h(\xi, \xi)+\beta h(\xi, \xi) g(X, Y) .
$$

Then adding the above two equations we get

$$
\beta h(X, Y)-\frac{\alpha}{2}[h(\phi X, Y)+h(X, \phi Y)]=\beta h(\xi, \xi) g(X, Y) .
$$

We see that $\beta h(X, Y)-\frac{\alpha}{2}[h(\phi X, Y)+h(X, \phi Y)]$ is a symmetric tensor of type $(0,2)$. Since $\mathcal{L}_{\xi} g(X, Y)$, $S(X, Y), \eta(X)=g(X, \xi)$ and $\eta(Y)=g(Y, \xi)$ are symmetric tensors of type $(0,2)$ and $\lambda, \mu$ are real constants, the sum $\mathcal{L}_{\xi} g(X, Y)+2 S(X, Y)+2 \mu \eta(X) \eta(Y)$ is a symmetric tensor of type $(0,2)$. 
Therefore, we can take the sum as an another symmetric tensor field of type $(0,2)$. Hence for we can assume that $\beta h(X, Y)-\frac{\alpha}{2}[h(\phi X, Y)+h(X, \phi Y)]=\mathcal{L}_{\xi} g(X, Y)+2 S(X, Y)+2 \mu \eta(X) \eta(Y)$.

Then we compute

$$
\beta h(\xi, \xi) g(X, Y)=\mathcal{L}_{\xi} g(X, Y)+2 \lambda g(X, Y)+2 \mu \eta(X) \eta(Y) .
$$

As $h$ is parallel so, $h(\xi, \xi)$ is constant. Hence, we can write $h(\xi, \xi)=-\frac{2}{\beta} \lambda$ where $\beta$ is constant and $\beta \neq 0$.

So, from the equation (3.7) we have

$$
\beta h(X, Y)-\frac{\alpha}{2}[h(\phi X, Y)+h(X, \phi Y)]=-2 \lambda g(X, Y),
$$

for any $X, Y \in \chi(M)$. Therefore $\mathcal{L}_{\xi} g(X, Y)+2 S(X, Y)+2 \mu \eta(X) \eta(Y)=-2 \lambda g(X, Y)$ and so $(g, \xi, \mu)$ becomes an $\eta$-Ricci soliton.

Corollary 3.2: Let $(M, g, \phi, \eta, \xi, \alpha, \beta)$ be a 3-dimensional trans-Sasakian manifold with $\alpha, \beta$ constants $(\beta \neq 0)$. If the symmetric $(0,2)$ tensor field $h$ admitting the condition $\beta h(X, Y)-$ $\frac{\alpha}{2}[h(\phi X, Y)+h(X, \phi Y)]=\mathcal{L}_{\xi} g(X, Y)+2 S(X, Y)$ is parallel with respect to the Levi-Civita connection associated to $\mathrm{g}$ with $\lambda=2 \mathrm{n}$. Then $(\mathrm{g}, \xi)$ becomes a Ricci soliton.

Next theorem shows the necessary condition for the existence of $\eta$-Ricci soliton on 3-dimensional trans-Sasakian manifolds.

Theorem 3.3: If 3-dimensional trans-Sasakian manifold satisfies an $\eta$-Ricci soliton then the manifold becomes $\eta$-Einstein manifold with $\alpha$ and $\beta$ constants.

Proof: From the equation (1.1) we get

$$
2 S(X, Y)=-g\left(\nabla_{X} \xi, Y\right)-g\left(X, \nabla_{Y} \xi\right)-2 \lambda g(X, Y)-2 \mu \eta(X) \eta(Y) .
$$

By using the equation (2.8) we get

$$
S(X, Y)=-(\beta+\lambda) g(X, Y)+(\beta-\mu) \eta(X) \eta(Y)
$$

and

$$
S(X, \xi)=-(\lambda+\mu) \eta(X) .
$$

Also from (2.11) we have

$$
\lambda+\mu=2\left(\beta^{2}-\alpha^{2}\right) .
$$

The Ricci operator $Q$ is defined by $g(Q X, Y)=S(X, Y)$. Then we get

$$
\mathrm{QX}=\left(\mu-\beta+2\left(\alpha^{2}-\beta^{2}\right)\right) X+(\beta-\mu) \eta(X) \xi .
$$


Then we can easily see that the manifold is an $\eta$-Einstein manifold.

We know a manifold is $\phi$-Ricci symmetric if $\phi^{2} \circ \nabla Q=0$. Now we prove the next theorem.

Theorem 3.4: If a $\phi$-Ricci symmetric trans-Sasakian manifold $(M, g)$ satisfies an $\eta$-Ricci soliton then $\mu=\beta, \lambda=2\left(\beta^{2}-\alpha^{2}\right)-\beta$ and $(M, g)$ is an Einstein manifold.

Proof: From the equation (3.13) we have

$$
\begin{gathered}
\left(\nabla_{X} Q\right) Y=\nabla_{X} Q Y-Q\left(\nabla_{X} Y\right) \\
=-\alpha(\beta-\mu) \eta(Y) \phi X+\beta(\beta-\mu) \eta(Y) X-(\beta-\mu) \eta(Y) \eta(X) \xi \\
+(\beta-\mu)[-\alpha g(\phi X, Y)+\beta g(\phi X, \phi Y)] \xi .
\end{gathered}
$$

Now applying $\phi^{2}$ both sides we have $\mu=\beta, \lambda=2\left(\beta^{2}-\alpha^{2}\right)-\beta$ and $(M, g)$ is an Einstein manifold.

We construct an example of $\eta$-Ricci soliton on 3-dimensional trans-Sasakian manifolds in the The next section.

\section{Example of $\eta$-Ricci solitons on 3-dimensional trans-Sasakian manifolds}

We consider the three dimensional manifold $M=\left\{(x, y, z) \in R^{3}: y \neq 0\right\}$ where $(x, y, z)$ are the standard coordinates in $R^{3}$. The vector fields

$$
e_{1}=e^{2 z} \frac{\partial}{\partial x}, e_{2}=e^{2 z} \frac{\partial}{\partial y}, e_{3}=\frac{\partial}{\partial z}
$$

are linearly independent at each point of $M$. Let $g$ be the Riemannian metric defined by

$$
g_{i j}= \begin{cases}1 & \text { for } \quad i=j \\ 0 & \text { for } \quad i \neq j .\end{cases}
$$

Let $\eta$ be the 1 -form defined by $\eta(Z)=g\left(Z, e_{3}\right)$ for any $Z \in \chi\left(M^{3}\right)$. Let $\phi$ be the $(1,1)$ tensor field defined by $\phi\left(e_{1}\right)=e_{2}, \phi\left(e_{2}\right)=-e_{1}, \phi\left(e_{3}\right)=0$. Then using the linearity property of $\phi$ and $g$ we have

$$
\eta\left(e_{2}\right)=1, \phi^{2}(Z)=-Z+\eta(Z) e_{2}, g(\phi Z, \phi W)=g(Z, W)-\eta(Z) \eta(W),
$$

for any $Z, W \in \chi\left(M^{3}\right)$. Thus for $e_{2}=\xi,(\phi, \xi, \eta, g)$ defines an almost contact metric structure on M. Now, after some calculation we have, 


$$
\left[e_{1}, e_{3}\right]=-2 e_{1},\left[e_{2}, e_{3}\right]=-2 e_{2},\left[e_{1}, e_{2}\right]=0 .
$$

The Riemannian connection $\nabla$ of the metric is given by the Koszul's formula which is

$$
2 g\left(\nabla_{X} Y, Z\right)=X g(Y, Z)+Y g(Z, X)-Z g(X, Y)-g(X,[Y, Z])-g(Y,[X, Z])+g(Z,[X, Y])
$$

By Koszul's formula we get,

$$
\begin{gathered}
\nabla_{e_{1}} e_{1}=2 e_{3}, \nabla_{e_{2}} e_{1}=0, \nabla_{e_{3}} e_{1}=0, \nabla_{e_{1}} e_{2}=0, \nabla_{e_{2}} e_{2}=2 e_{3}, \\
\nabla_{e_{3}} e_{2}=0, \nabla_{e_{1}} e_{3}=-2 e_{1}, \nabla_{e_{2}} e_{3}=-2 e_{2}, \nabla_{e_{3}} e_{3}=0 .
\end{gathered}
$$

From the above it can be easily shown that $M^{3}(\phi, \xi, \eta, g)$ is a trans-Sasakian manifold of type $(0,-2)$.

Here

$$
\begin{gathered}
R\left(e_{1}, e_{2}\right) e_{2}=-4 e_{1}, R\left(e_{3}, e_{2}\right) e_{2}=4 e_{2}, R\left(e_{1}, e_{3}\right) e_{3}=-4 e_{1}, R\left(e_{2}, e_{3}\right) e_{3}=-4 e_{2}, \\
R\left(e_{3}, e_{1}\right) e_{1}=-4 e_{2}, R\left(e_{2}, e_{1}\right) e_{1}=4 e_{3} .
\end{gathered}
$$

So, we have

$$
S\left(e_{1}, e_{1}\right)=0, S\left(e_{2}, e_{2}\right)=0, S\left(e_{3}, e_{3}\right)=-8 .
$$

From the equation (1.1) we get $\lambda=-2$ and $\mu=6$. Therefore, $(g, \xi, \lambda, \mu)$ is an $\eta$-Ricci soliton on $M^{3}(\phi, \xi, \eta, g)$.

In the next sections we consider $\eta$-Ricci Solitons on 3-dimensional trans-Sasakian manifolds satisfying some curvature conditions.

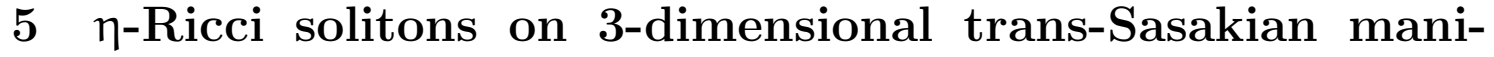 folds satisfying $R(\xi, X) \cdot S=0$}

First we suppose that 3-dimensional trans-Sasakian manifolds with $\eta$-Ricci solitons satisfy the condition

$$
R(\xi, X) \cdot S=0 .
$$

Then we have

$$
S(R(\xi, X) Y, Z)+S(Y, R(\xi, X) Z)=0
$$


for any $X, Y, Z \in \chi(M)$.

Using the equations (2.12), (3.10), (3.11) we get

$$
(\beta-\mu) g(X, Y) \eta(Z)+(\beta-\mu) g(X, Z) \eta(Y)-2(\beta-\mu) \eta(X) \eta(Y) \eta(Z)=0 .
$$

Put $Z=\xi$ we have

$$
(\beta-\mu) g(X, Y)-(\beta-\mu) \eta(X) \eta(Y)=0 .
$$

Setting $X=\phi X$ and $Y=\phi Y$ in the above equation we get

$$
(\beta-\mu) g(\phi X, \phi Y)=0 .
$$

Again using the equation (3.12) we have

$$
\mu=\beta, \lambda=2\left(\beta^{2}-\alpha^{2}\right)-\beta .
$$

Also we can easily see that $M$ is an Einstein manifold. So we have the following theorem.

Theorem 5.1: If a 3 -dimensional trans-Sasakian manifold $(M, g, \phi, \eta, \xi, \alpha, \beta)$ with $\alpha, \beta$ constants admitting an $\eta$-Ricci soliton satisfies the condition $R(\xi, X) \cdot S=0$ then $\mu=\beta, \lambda=2\left(\beta^{2}-\alpha^{2}\right)-\beta$ and $M$ is an Einstein manifold.

Corollary 5.2: A 3 -dimensional trans-Sasakian manifold with $\alpha, \beta$ constants satisfies the condition $R(\xi, X) \cdot S=0$, there is no Ricci soliton with the potential vector field $\xi$.

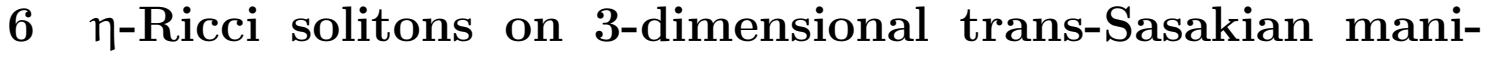 folds satisfying $S(\xi, X) \cdot R=0$}

We consider 3-dimensional trans-Sasakian manifolds with $\eta$-Ricci solitons satisfying the condition

$$
S(\xi, X) \cdot R=0 .
$$


So we have

$$
\begin{gathered}
S(X, R(Y, Z) W) \xi-S(\xi, R(Y, Z) W) X+S(X, Y) R(\xi, Z) W-S(\xi, Y) R(X, Z) W \\
+S(X, Z) R(Y, \xi) W-S(\xi, Z) R(Y, X) W+S(X, W) R(Y, Z) \xi-S(\xi, W) R(Y, Z) X=0 .
\end{gathered}
$$

Taking inner product with $\xi$ then the above equation becomes

$$
\begin{gathered}
S(X, R(Y, Z) W)-S(\xi, R(Y, Z) W) \eta(X)+S(X, Y) \eta(R(\xi, Z) W) \\
-S(\xi, Y) \eta(R(X, Z) W)+S(X, Z) \eta(R(Y, \xi) W)-S(\xi, Z) \eta(R(Y, X) W) \\
+S(X, W) \eta(R(Y, Z) \xi)-S(\xi, W) \eta(R(Y, Z) X)=0 .
\end{gathered}
$$

Put $W=\xi$ and using the equations (2.10), (2.12), (3.10), (3.11) we get

$$
-(\beta+\lambda) g(X, R(Y, Z) \xi)+(\lambda+\mu) \eta(R(Y, Z) X)=0 .
$$

Also we have

$$
\eta(R(Y, Z) X)=-g(X, R(Y, Z) \xi) .
$$

So from the equation (6.2) we get

$$
(\beta+2 \lambda+\mu) g(X, R(Y, Z) \xi)=0 .
$$

Again using the equation (3.12) we have

$$
\mu=\beta+4\left(\beta^{2}-\alpha^{2}\right), \quad \lambda=-\left[2\left(\beta^{2}-\alpha^{2}\right)+\beta\right] .
$$

So we have the following theorem.

Theorem 6.1: If a 3 -dimensional trans-Sasakian manifold $(M, g, \phi, \eta, \xi, \alpha, \beta)$ with $\alpha, \beta$ constants admitting an $\eta$-Ricci soliton satisfies the condition $S(\xi, X) \cdot R=0$ then $\mu=\beta+4\left(\beta^{2}-\alpha^{2}\right), \quad \lambda=$ $-\left[2\left(\beta^{2}-\alpha^{2}\right)+\beta\right]$. 
Corollary 6.2: A 3-dimensional trans-Sasakian manifold with $\alpha, \beta$ constants satisfies the condition $S(\xi, X) \cdot R=0$, there is no Ricci soliton with the potential vector field $\xi$.

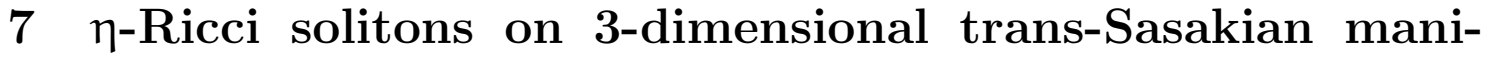 folds satisfying $W_{2}(\xi, X) \cdot S=0$}

Definition 7.1. Let $M$ be 3-dimensional trans-Sasakian manifold with respect to semi-Symmetric metric connection. The $W_{2}$-curvature tensor of $M$ is defined by 16

$$
W_{2}(X, Y) Z=R(X, Y) Z+\frac{1}{2}(g(X, Z) Q Y-g(Y, Z) Q X) .
$$

We assume 3-dimensional trans-Sasakian manifolds with $\eta$-Ricci solitons satisfying the condition

$$
W_{2}(\xi, X) \cdot S=0 .
$$

Then we have

$$
\mathrm{S}\left(\mathrm{W}_{2}(\xi, X) \mathrm{Y}, \mathrm{Z}\right)+\mathrm{S}\left(\mathrm{Y}, \mathrm{W}_{2}(\xi, X) Z\right)=0
$$

for any $X, Y, Z \in \chi(M)$.

Using the equations (2.12), (3.10), (3.11), (7.1) we get

$$
\begin{aligned}
& {\left[-\frac{(\beta+\lambda)}{2}(\lambda+\mu)+\frac{(\beta+\lambda)^{2}}{2}+(\beta-\mu)\left(\alpha^{2}-\beta^{2}\right)+(\lambda+\mu) \frac{(\beta-\mu)}{2}\right] g(X, Y) \eta(Z)} \\
& +\left[\frac{(\beta+\lambda)^{2}}{2}-\frac{(\beta+\lambda)}{2}(\lambda+\mu)+(\beta-\mu)\left(\alpha^{2}-\beta^{2}\right)+(\lambda+\mu) \frac{(\beta-\mu)}{2}\right] g(X, Z) \eta(Y) \\
& +\left[-(\beta+\lambda)(\beta-\mu)-2(\beta-\mu)\left(\alpha^{2}-\beta^{2}\right)-(\beta-\mu)(\lambda+\mu)\right] \eta(X) \eta(Y) \eta(Z)=0 .
\end{aligned}
$$

Put $Z=\xi$ in the above equation we get

$$
\begin{gathered}
{\left[-\frac{(\beta+\lambda)}{2}(\lambda+\mu)+\frac{(\beta+\lambda)^{2}}{2}+(\beta-\mu)\left(\alpha^{2}-\beta^{2}\right)+(\lambda+\mu) \frac{(\beta-\mu)}{2}\right] g(X, Y)} \\
+\left[\frac{(\beta+\lambda)^{2}}{2}-\frac{(\beta+\lambda)}{2}(\lambda+\mu)+(\beta-\mu)\left(\alpha^{2}-\beta^{2}\right)+(\lambda+\mu) \frac{(\beta-\mu)}{2}\right.
\end{gathered}
$$




$$
\left.-(\beta+\lambda)(\beta-\mu)-2(\beta-\mu)\left(\alpha^{2}-\beta^{2}\right)-(\beta-\mu)(\lambda+\mu)\right] \eta(X) \eta(Y)=0 .
$$

Setting $X=\phi X$ and $Y=\phi Y$ in the above equation we get

$$
(\beta-\mu)\left(\frac{\left(\beta+2 \lambda+\mu+2\left(\alpha^{2}-\beta^{2}\right)\right)}{2}\right) g(\phi X, \phi Y)=0 .
$$

Again using the equation (3.12) we have

$$
\mu=\beta, \lambda=2\left(\beta^{2}-\alpha^{2}\right)-\beta
$$

or

$$
\mu=2\left(\beta^{2}-\alpha^{2}\right)+\beta, \quad \lambda=-\beta .
$$

So we have the following theorem.

Theorem 7.1: If a 3 -dimensional trans-Sasakian manifold $(M, g, \phi, \eta, \xi, \alpha, \beta)$ with $\alpha, \beta$ constants admitting an $\eta$-Ricci soliton satisfies the condition $W_{2}(\xi, X) \cdot S=0$ then $\mu=\beta, \lambda=2\left(\beta^{2}-\alpha^{2}\right)-\beta$ or $\mu=2\left(\beta^{2}-\alpha^{2}\right)+\beta, \lambda=-\beta$.

Corollary 7.2: A 3-dimensional trans-Sasakian manifold with $\alpha, \beta$ constants satisfies the condition $W_{2}(\xi, X) \cdot S=0$, there is no Ricci soliton with the potential vector field $\xi$.

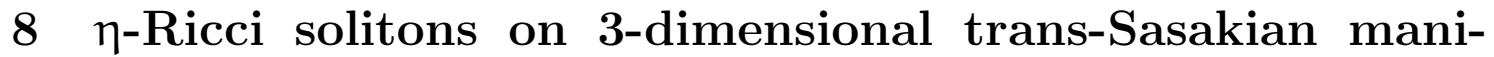 folds satisfying $S(\xi, X) \cdot W_{2}=0$}

Suppose that 3-dimensional trans-Sasakian manifolds with $\eta$-Ricci solitons satisfy the condition

$$
S(\xi, X) \cdot W_{2}=0
$$

So we have

$$
\begin{gathered}
S\left(X, W_{2}(Y, Z) V\right) \xi-S\left(\xi, W_{2}(Y, Z) V\right) X+S(X, Y) W_{2}(\xi, Z) V-S(\xi, Y) W_{2}(X, Z) V \\
+S(X, Z) W_{2}(Y, \xi) V-S(\xi, Z) W_{2}(Y, X) V+S(X, V) W_{2}(Y, Z) \xi-S(\xi, V) W_{2}(Y, Z) X=0 .
\end{gathered}
$$


Taking inner product with $\xi$ then the above equation becomes

$$
\begin{gathered}
S\left(X, W_{2}(Y, Z) V\right)-S\left(\xi, W_{2}(Y, Z) V\right) \eta(X)+S(X, Y) \eta\left(W_{2}(\xi, Z) V\right) \\
-S(\xi, Y) \eta\left(W_{2}(X, Z) V\right)+S(X, Z) \eta\left(W_{2}(Y, \xi) V\right)-S(\xi, Z) \eta\left(W_{2}(Y, X) V\right) \\
+S(X, V) \eta\left(W_{2}(Y, Z) \xi\right)-S(\xi, V) \eta\left(W_{2}(Y, Z) X\right)=0 .
\end{gathered}
$$

Put $V=\xi$ and using the equations (2.10), (2.12), (3.10), (3.11), (7.1) we get

$$
-(\beta+\lambda) g\left(X, W_{2}(Y, Z) \xi\right)+(\lambda+\mu) \eta\left(W_{2}(Y, Z) X\right)=0 .
$$

Using the equations (3.10), (3.11), (7.1) then the equation (8.2) becomes

$$
\left[(\beta+\lambda)^{2}+(\lambda+\mu)^{2}+2\left(\alpha^{2}-\beta^{2}\right)(\beta+2 \lambda+\mu)\right] g(X, R(Y, Z) \xi)=0 .
$$

Using the equation (3.12) we have

$$
\mu=\beta, \quad \lambda=2\left(\beta^{2}-\alpha^{2}\right)-\beta
$$

or

$$
\mu=2\left(\beta^{2}-\alpha^{2}\right)+\beta, \lambda=-\beta .
$$

So we have the following theorem.

Theorem 8.1: If Let a 3 -dimensional trans-Sasakian manifold $(M, g, \phi, \eta, \xi, \alpha, \beta)$ with $\alpha, \beta$ constants admitting an $\eta$-Ricci soliton satisfies the condition $S(\xi, X) \cdot W_{2}=0$ then $\mu=\beta, \quad \lambda=$ $2\left(\beta^{2}-\alpha^{2}\right)-\beta$ or $\mu=2\left(\beta^{2}-\alpha^{2}\right)+\beta, \lambda=-\beta$.

Corollary 8.2: A 3-dimensional trans-Sasakian manifold with $\alpha, \beta$ constants satisfies the condition $S(\xi, X) \cdot W_{2}=0$, there is no Ricci soliton with the potential vector field $\xi$.

Acknowledgement: The author wish to express her sincere thanks and gratitude to the referee for valuable suggestions towards the improvement of the paper. 


\section{References}

[1] C. S. Bagewadi, G. Ingalahalli, S. R. Ashoka, A stuy on Ricci solitons in Kenmotsu Manifolds, ISRN Geometry, (2013), Article ID 412593, 6 pages.

[2] A. Bhattacharyya, T. Dutta, and S. Pahan, Ricci Soliton, Conformal Ricci Soliton And Torqued Vector Fields, Bulletin of the Transilvania University of Brasov Series III: Mathematics, Informatics, Physics,, Vol 10(59), No. 1 (2017), 39-52.

[3] A. M. Blaga, Eta-Ricci solitons on para-Kenmotsu manifolds, Balkan Journal of Geometry and Its Applications, Vol.20, No.1, 2015, pp. 1-13.

[4] C. Călin, M. Crasmareanu, Eta-Ricci solitons on Hopf hypersurfaces in complex forms, Revue Roumaine de Math. Pures et app., 57 (1), (2012), 53-63.

[5] B. Y. Chen, S. Deshmukh, Geometry of compact shrinking Ricci solitons, Balkan Journal of Geometry and Its Applications, Vol.19, No.1, 2014, pp. 13-21

[6] J.C. Cho, M. Kimura Ricci solitons and real hypersurfaces in a complex space form, Tohoku Math. J. 61 (2), (2009), 205-2012.

[7] O. Chodosh, F. T.-H Fong, Rational symmetry of conical Kähler-Ricci solitons, Math. Ann., 364(2016), 777-792.

[8] A. Futaki, H. Ono, G. Wang, Transverse Kähler geometry of Sasaki manifolds and toric Sasaki-Einstein manifolds, J. Diff. Geom. 83 (3), (2009), 585-636.

[9] S. Golab, On semi-symmetric and quarter-symmetric linear connection, Tensor. N. S., 29(1975), 249-254.

[10] R. S. Hamilton, The formation of singularities in the Ricci flow, Surveys in Differential Geometry (Cambridge, MA, 1993), 2, 7-136, International Press, Combridge, MA, 1995.

[11] R. S. Hamilton, The Ricci flow on surfaces, Mathematical and general relativity, Contemp. math, 71(1988), 237-261.

[12] G. Ingalahalli, C. S. Bagewadi, Ricci solitons on $\alpha$-Sasakian Manifolds, ISRN Geometry, (2012), Article ID 421384, 13 pages.

[13] J. C. Marrero, Ihe local structure of trans-Sasakian manifolds, Ann. Mat. Pura. Appl., (4), 162(1992), 77-86.

[14] J. Morgan, G. Tian, Ricci Flow and the Poincaŕe Conjecture, American Mathematical Society Clay Mathematics Institute, (2007). 
[15] J. A. Oubina, New classes of almost contact metric structures, pub. Math. Debrecen, 20 (1), (2015), 1-13.

[16] G. P. Pokhariyal, R. S. Mishra, The curvature tensors and their relativistic significance, Yokohama Math. J., 18(1970), 105-106.

[17] D. G. Prakasha, B. S. Hadimani, $\eta$-Ricci solitons on para-Sasakian manifolds, Journal of Geometry, (2016), DOI: 10.1007/s00022-016-0345-z, pp 1-10. 